\title{
Gustatory Function in Patients With Chronic Rhinosinusitis
}

\author{
Axel Wolf, MD' (iD, Bertold Renner, MD², Peter V. Tomazic, MD', \\ and Christian A. Mueller, MD ${ }^{3}$
}

\begin{abstract}
Objectives: Recent research has shown that taste receptors in airway epithelial cells are involved in defending against upper respiratory tract infection. The aim of the present study was to investigate gustatory function in patients with chronic rhinosinusitis (CRS).

Methods: Taste function was assessed using the extended "taste strip" test in 37 patients with CRS (20 males, I7 females; mean age $=32.1$ years; range, $20-82$ years) and 135 healthy controls (70 males, 65 females; mean age = 29.5 years; range, 18-84 years).

Results: The mean $( \pm S D)$ total extended taste score was $12.8( \pm 3.5)$ in patients and $14.5( \pm 3.2)$ in controls. Analysis of variance indicated an interaction of sinusitis and gender $(P<.05)$ with significantly lower total scores and significantly poorer results for the bitter taste among male patients compared to controls $(P<.0 \mathrm{I})$. In addition, CRS patients exhibited a trend toward decreased sweet taste perception compared to controls, but this did not reach significance $(P=.05 \mathrm{I})$.

Conclusions: Patients with CRS exhibited decreased gustatory function compared to healthy controls. The effect was most pronounced for bitter taste. Thus, the assessment of gustatory function seems to be useful for detecting potential risk factors for recurrent upper respiratory tract infection.
\end{abstract}

\section{Keywords}

chemosensory, gustation, strips, supertaster, bitter, sweet

\section{Introduction}

Chronic rhinosinusitis (CRS) is a chronic inflammatory disease of the upper airway tract that involves the mucosa of the nasal cavity and nasal sinuses. Chronic rhinosinusitis is influenced by mucociliary function, anatomy, multifactorial infection, immune response, and allergy. The roles of these and other factors and their impact on the pathophysiology of the disease are still being investigated. ${ }^{1-3}$ Recent studies have shown that taste receptors in airway epithelial cells are involved in the immune defense of the upper respiratory tract. A variety of pathways influence mucociliary clearance, local immune response, and the recruitment of immune cells. Thus, taste receptors may play a role in the pathophysiology of chronic upper airway inflammation, such as CRS, with or without nasal polyps (CRSwNP and CRSsNP, respectively). ${ }^{2,4-10}$

The stimulation of bitter taste receptors has a positive effect on mucociliary clearance and directs local bactericidal defense. ${ }^{1-3,8}$ Various bitter taste receptor isoforms in the T2R family have been investigated over the past few decades; the T2R38 taste receptor has been studied because of its expression in epithelial cells throughout the upper respiratory tract. Genetic variations influencing an individual's sensitivity to the bitter taste may affect susceptibility to infection and lead to CRS. ${ }^{2,4-8,10,11}$

The "taste strip" test is a validated tool for the assessment of gustatory function. ${ }^{4,11-15}$ The extended version of the test consists of 24 taste strips comprising 6 concentrations of each taste: sweet, sour, salty, and bitter. Compared to other gustatory tests, the extended taste strips test permits a more detailed assessment of gustatory function without a ceiling effect. ${ }^{16}$ Assessment of the taste acuity in patients with CRS would provide detailed information about the impact of taste receptors on recurrent upper airway

\footnotetext{
'Medical University of Graz, Department of Otothinolaryngology, Head and Neck Surgery, Graz, Austria

${ }^{2}$ University of Erlangen-Nuernberg, Institute of Experimental and Clinical Pharmacology and Toxicology, Erlangen, Germany

${ }^{3}$ Medical University Vienna, Department of Otorhinolaryngology, Vienna, Austria
}

\section{Corresponding Author:}

Christian A. Mueller, Department of Otorhinolaryngology, Medical University Vienna, Waehringer Guertel 18-20, 1090 Wien, Austria.

Email: christian.a.mueller@meduniwien.ac.at 
infections. Thus, the present study investigated gustatory function with a clinical test in patients with CRS compared to healthy controls.

\section{Materials and Methods}

\section{Ethics}

This study was performed according to the guidelines of the Declaration of Helsinki on biomedical research involving human subjects. The study was approved by the local ethics committees, and all subjects provided written informed consent.

\section{Subjects}

The study included 37 patients with CRS (17 females, 20 males; mean age \pm standard deviation $[\mathrm{SD}]=32.1 \pm 13.8$ years; range, 20-82 years) diagnosed based on current guidelines. ${ }^{2}$ Patients with tumors, dentogenic sinusitis, or fungal infection were excluded from the study. Twenty-four patients had CRSsNP, and 13 had CRSwNP based on interviews, clinical examination, and computed tomography (CT) scans. Functional endoscopic sinus surgery (FESS) was performed in all patients before or after inclusion. Interviews revealed that 7 patients had allergies, but only 1 took permanent medication (desloratadine). Six patients took permanent medication for other diseases (ramipril, simvastatin, lisinopril, urapidil, atorvastatin, amlodipine, montelukast, or bisoprolol). No patient experienced dysgeusia due to medication. Thirty-one patients took no medication for at least 2 weeks prior to testing.

The healthy control group for comparison purposes comprised 70 males and 65 females (mean age $=29.5 \pm$ 12.5 years; range, $18-84$ years).

\section{Measurements}

Subjective gustatory and olfactory function were evaluated on 10-point scales before testing $(1=$ no taste or olfaction, $10=$ excellent taste or olfaction); gustatory function was assessed before surgery or at least 6 months after surgery. Each patient was tested with 24 taste strips in the extended version of the taste strip test. The filter paper strips consisted of 6 concentrations of each of the following: sweet (sucrose), sour (citric acid), salty (sodium chloride), and bitter (quinine hydrochloride) ${ }^{16}$ Subjects did not eat, drink, or smoke at least 1 hour prior to the test.

Testing started with the lowest concentrations. The subjects were asked to respond to a list of 5 possible answers (sweet, sour, salty, bitter, no taste) as taste detection (threshold) could be separated from taste identification (recognition) by using a non-forced choice procedure. After the presentation of each taste strip, the subject took a sip of tap water.
Two taste scores were calculated. The first was the extended taste score identification (ETS-I) or the original extended taste score ${ }^{16}$ which is based on the identification of taste using a non-forced choice paradigm: Each correct answer was given 1 point, and incorrectly identified taste qualities were given no points.

A non-forced choice procedure was also used to calculate the second score, the extended taste score identification/perception (ETS-IP), but each correct answer was given 1 point and each perceived but incorrectly identified taste quality 0.5 points. Both scores ranged from 0 to 24 points.

\section{Statistical Analysis}

IBM SPSS 23.0 (Chicago, Illinois, USA) was used for statistical analyses. For an exploratory comparison of mean taste scores, we performed unpaired 2-sample $t$ tests without adjusting for multiple comparisons. The impact of potential factors on taste scores was determined by a general linear model (GLM) and univariate analysis of variance. For post hoc testing, unpaired $t$ tests or MannWhitney tests were used, as appropriate. Correlation analyses were performed with the Pearson's test. The alpha level was set to .05 .

\section{Results}

On 10-point scales, the mean \pm SD subjective values were $6.34 \pm 2.24$ for taste and $5.21 \pm 2.76$ for olfactory function. An overall correlation analysis revealed no significant correlation of ETS-I with subjective taste $(r=-0.32, P=.858)$ or olfactory function $(r=0.106, P=.556)$. Exploratory $t$ test revealed lower taste scores in CRS patients than controls (Table 1), and the GLM for all taste qualities revealed a significant overall effect of age and gender (ETS-I age: $P<.001, d f=6.154, F=7.08$; gender: $P=.006, d f=6.154$, $F=3.16$; ETS-IP age: $P<.001, d f=4.156, F=10.03$; gender: $P=.041, d f=0.156, F=2.55)$. Smoking habits and medication did not exert any consistent overall effects. In addition, the total score (ETS-I and ETS-IP) exhibited significant age and gender effects (age: $P<.001, d f=1.167$, $F=31.46$ and $F=30.53$; gender: $P<.01, d f=1.167, F=13.12$ and $F=11.76$ ), with lower scores for men than women. We also observed an association between gender and sinusitis $(P=.040, d f=1.167, F=4.28)$, with a greater reduction in male patients than controls $(P=.002$; Figure 1A). A corresponding effect was observed in the total ETS-IP score (age: $P<.001, d f=1.167, F=30.53$; gender: $P=.001$, $d f=1.167, F=11.76)$, with an interaction between age and sinusitis $(P=.019, d f=1.167, F=5.58)$ and lower scores in men compared to women $(P=.001$; Figure 1B).

The ETS-IP scores for the sweet taste revealed a tendency toward lower taste scores in CRS patients than 
Table I. Extended Taste Scores in Patients and Controls. ${ }^{a}$

\begin{tabular}{|c|c|c|c|c|c|}
\hline & \multicolumn{2}{|c|}{ Patients $(\mathrm{n}=37)$} & \multicolumn{2}{|c|}{ Control Group $(n=135)$} & $P$ Value \\
\hline Total & 12.85 & $(3.52)$ & 14.47 & $(3.18)$ & .005 \\
\hline Sweet & 3.84 & $(1.23)$ & 4.54 & $(1.22)$ & .002 \\
\hline Sour & 2.46 & $(1.24)$ & 2.80 & $(1.0)$ & .068 \\
\hline Salty & 3.62 & $(1.26)$ & 3.97 & $(1.31)$ & .150 \\
\hline Bitter & 2.95 & $(1.68)$ & 3.15 & $(1.16)$ & .389 \\
\hline \multicolumn{6}{|l|}{ ETS-IP } \\
\hline Total & 14.78 & $(3.50)$ & 16.52 & $(2.86)$ & .006 \\
\hline Sweet & 4.15 & $(1.10)$ & 4.80 & $(1.06)$ & .005 \\
\hline Sour & 3.15 & (1.01) & 3.55 & $(0.91)$ & .022 \\
\hline Salty & 4.15 & $(1.05)$ & 4.61 & $(0.96)$ & .015 \\
\hline Bitter & 3.36 & $(1.56)$ & 3.54 & $(1.08)$ & .530 \\
\hline
\end{tabular}

${ }^{a}$ Data are presented as mean (SD). The maximum score for the extended taste strip test was 6 for each taste quality and 24 for the total score. $P$ values were determined by $t$ test of group differences without adjustment for multiple comparisons. ETS-I, extended taste score-identification; ETS-IP, extended taste score-identification/perception.

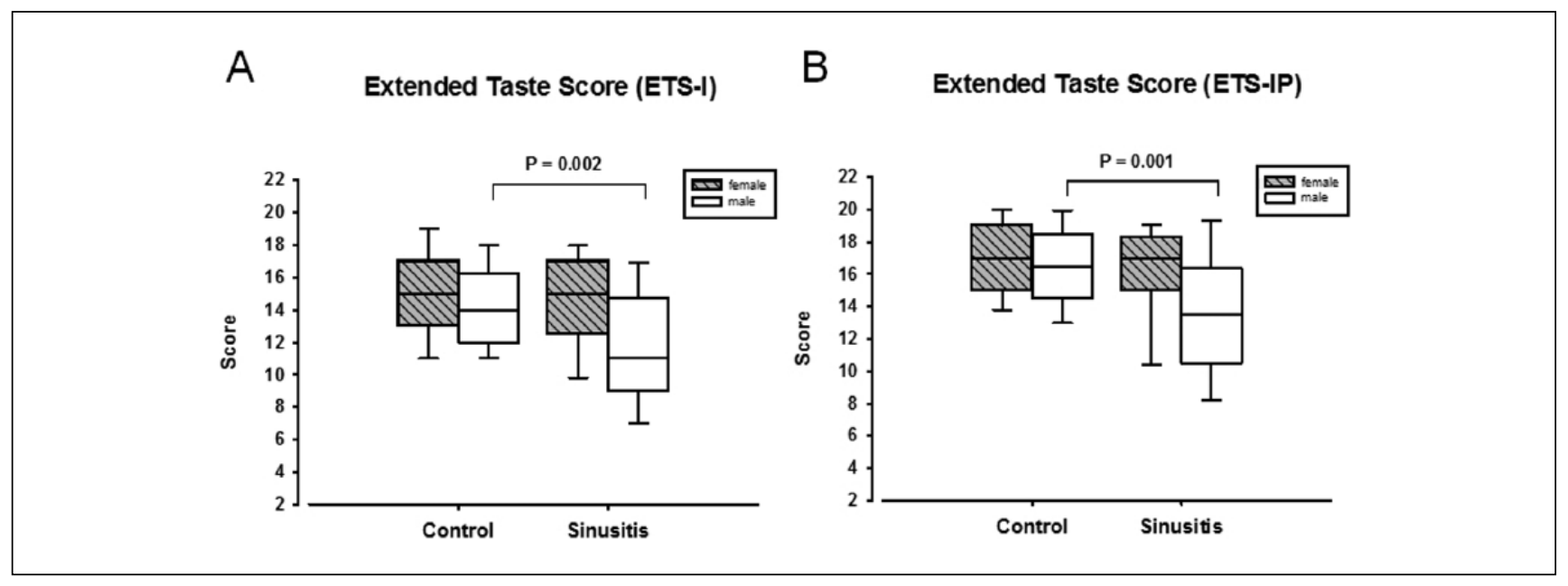

Figure I. Total taste scores in patients with chronic rhinosinusitis (sinusitis). Significantly lower total scores were obtained in male patients compared to controls. (A) ETS-I for identification. (B) ETS-IP for identification and perception. ETS-I, extended taste scoreidentification; ETS-IP, extended taste score-identification/perception. $P \leq .002, t$ test.

controls for both genders (sinusitis vs control: $P=.051$, $d f=1.167, F=3.85$; age: $P<.001, d f=1.167, F=17.49$; gender: $P=.018, d f=1.167, F=5.71$; Figure 2). Scores for bitter taste revealed a similar effect as with the total score. A significant age and gender effect (ETS-I and ETS-IP age: $P \leq .005, d f=1.167, F=8.12$; ETS-I and ETS-IP gender: $P<.001, d f=1.167, F=24.45)$ and an interaction between sinusitis and gender (ETS-I and ETS-IP: $P<.001, d f=1.167, F=14.40$ ) were observed. Post hoc analysis showed impaired bitter taste function in male CRS patients (Mann-Whitney tests: ETS-I $P=.003$ and ETS-IP $P=.004$; Figures $3 \mathrm{~A}$ and $3 \mathrm{~B}$ ).

A subgroup analysis with controls matched for age, gender, smoking habits, and medication was performed to confirm the data. Gender and age were significant factors with regard to taste scores. A significant interaction was noted between gender and sinusitis in the ETS-I/IP bitter score $(P$ $<.01)$ and ETS-IP total score $(P<.05)$.

Another subgroup analysis was performed to investigate the impact of nasal polyps on the overall results. The total scores were not significantly different for CRSwNP patients and controls, but the interaction of gender and sinusitis was still significant for the bitter taste on the ETS-I and ETS-IP $(P<.05)$.

\section{Discussion}

The present study revealed impaired taste function in men with CRS. The recently validated extended taste strips test was used with 6 concentrations instead of $4 .^{12,16}$ Though the extended taste score is based on a non-forced choice paradigm, some authors prefer forced-choice procedures. One 
Extended Taste Score Sweet (ETS-IP)

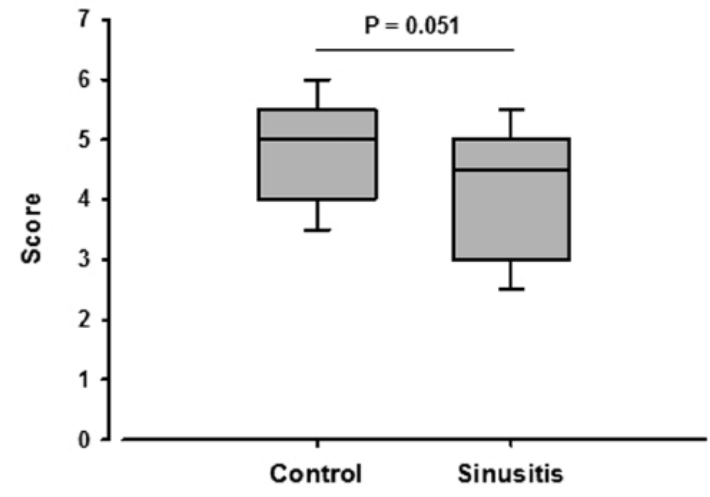

Figure 2. Sweet taste score results in patients with chronic rhinosinusitis (sinusitis). The combined taste score (ETS-IP) tended to be lower in the CRS (sinusitis) group but did not reach significance $(P=.05 \mathrm{I})$. ETS-I, extended taste scoreidentification; ETS-IP, extended taste score-identification/ perception.

major advantage of the forced-choice test is that it eliminates the subject's response bias. ${ }^{17}$ In our investigation, the use of a non-forced choice model permitted the perception of taste to be distinguished from the correct identification of taste. We determined an additional taste score (ETS-IP) that registered mismatched but perceived tastes, which made it possible to extract additional information from the subjects' answers. Moreover, the ETS-I results were confirmed. This approach confirmed the validity of a non-forced choice procedure, provided that a forced-choice procedure is not needed for other purposes, such as litigation.

Men with CRS in the overall patient group and CRSwNP subgroup had lower ETS-I scores and impaired bitter taste function compared to healthy controls after adjusting for sex and age. The taste strips used here included quinine. No investigation has yet assessed potential variations in bitter perception by quinine, although recent work has shown its association with allelic bitter receptor variations. ${ }^{18}$ Our findings confirm published reports of the influence of taste receptors on local immune defense in the clinical setting. ${ }^{3-8}$ In particular, the T2R38 bitter taste receptors and sweet taste receptors play a role in the local immune response in the upper airway tract. Stimulation of bitter taste receptors influences the local production of nitrogen monoxide and intracellular calcium release, enhancing mucociliary clearance, the recruitment of professional immune cells, and the local immune response. ${ }^{3-8,10}$ Persons with certain T2R38 receptor alleles that enhance receptor sensitivity are thought to be less susceptible to CRS and respond better to medical treatment than those with other alleles. ${ }^{3-8}$ Recently, a correlation was suggested between T2R38 function and nasal symptoms, such as colds, allergy, and overall nasal quality of life, in healthy patients. ${ }^{9}$ Accordingly, we observed reduced taste function in CRS patients compared to healthy controls. This effect was gender-specific and more pronounced in the male subgroup.

Analysis of variance revealed a significant influence of age on taste scores in the overall patient group and CRSwNP subgroup, and we were able to confirm decreased gustatory perception in older patients. ${ }^{12,17-20}$ Furthermore, the analysis of variance confirmed higher taste scores in women than in men. ${ }^{12,17-20}$ Decreased sweet taste performance was observed among patients after adjusting for age, but it was not significant. Recently, Lee et $\mathrm{al}^{21}$ suggested that sweet and bitter receptors in the upper respiratory tract play an antagonistic role in local immune defense. The bitter taste receptor-mediated local immune response is antagonized by the activation of sweet receptors. ${ }^{21}$ In the case of bacterial infection, the concentration of glucose decreases, leading to a release of sweet taste-mediated inhibition of bitter taste-mediated immune defense. Workman et $\mathrm{al}^{22}$ recently reported significantly higher sweet taste intensity ratings in patients with CRS than controls. Conversely, patients rated the bitter substance denatonium as having a lower intensity. Similar effects on sweet taste receptor signaling at a cellular level were described by Lee et $\mathrm{al}^{23}$ and bacterial $\mathrm{D}$-amino acids were shown to suppress the bitter taste receptor-mediated immune response by activating sweet taste receptors.

The results of this study with regard to sweet taste do not necessarily disagree with published data. First, central taste performance as measured by taste tests may not always reflect peripheral taste sensitivity at the receptor level. As such, sweet taste sensitivity may be normal or even enhanced in our subgroup, but central processing may be different compared to the control group. Second, no reports are available on the peripheral sweet taste sensitivity in quinine bitter taste deficiency. Future studies may show unaltered, or even decreased, peripheral sensitivity in this subgroup. However, the relatively small sample size was not adequate to test this hypothesis in the present study.

In agreement with previous studies, we found no significant correlation between self-assessed gustatory or olfactory function and measured taste acuity. ${ }^{12-15,24,25}$ This underlines the necessity of validated tests of chemosensory function. In the present study, differences in taste acuity, as measured by decreased bitter detection, were assessed in male patients with CRS compared to controls using a clinical taste test. These results may reflect the status of genetic variation in bitter taste receptors at a clinical level and the usefulness of testing taste function in patients with CRS. 


\section{A}

Extended Taste Score Bitter (ETS-I)

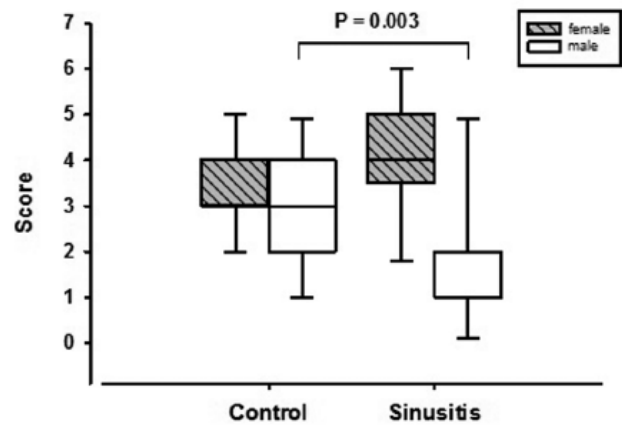

B

\section{Extended Taste Score Bitter (ETS-IP)}

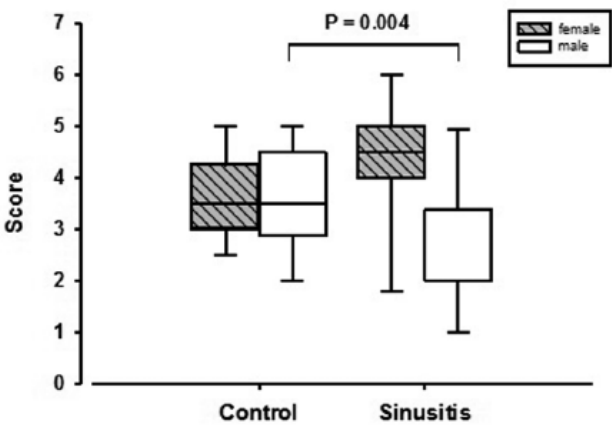

Figure 3. Bitter taste scores in patients with chronic rhinosinusitis (sinusitis). Significantly lower bitter taste scores were obtained in male patients compared to controls. (A) ETS-I for identification. (B) ETS-IP for identification and perception. ETS-I, extended taste score-identification; ETS-IP, extended taste score-identification/perception. $P \leq .004$, Mann-Whitney test.

\section{Declaration of Conflicting Interests}

The author(s) declared no potential conflicts of interest with respect to the research, authorship, and/or publication of this article.

\section{Funding}

The author(s) disclosed receipt of the following financial support for the research, authorship, and/or publication of this article: This study was approved by the local ethics committee (approval numbers 1014/2013 and 1345/2015).

\section{ORCID iD}

Axel Wolf (iD https://orcid.org/0000-0003-0086-5840

\section{References}

1. Henkin RI, Gill JR, Bartter FC. Studies on taste thresholds in normal man and in patients with adrenal cortical insufficiency: the role of adrenal cortical steroids and of serum sodium concentration. J Clin Invest. 1963;42:727-735.

2. Fokkens WJ, Lund VJ, Mullol J, et al. European position paper on rhinosinusitis and nasal polyps 2012. Rhinol Suppl. 2012;1-298.

3. Adappa ND, Howland TJ, Palmer JN, et al Genetics of the taste receptor T2R38 correlates with chronic rhinosinusitis necessitating surgical intervention. Int Forum Allergy Rhinol. 2013;3:184-187.

4. Reed DR. Birth of a new breed of supertaster. Chemical Senses. 2008;33(6):489-491.

5. Cohen SP, Buckley BK, Kosloff M, et al. Regulator of G-protein signaling-21 (RGS21) is an inhibitor of bitter gustatory signaling found in lingual and airway epithelia. $J$ Biol Chem. 2012;287:41706-41719.

6. Lee RJ, Xiong G, Kofonow JM, et al. T2R38 taste receptor polymorphisms underlie susceptibility to upper respiratory infection. J Clin Invest. 2012;122:4145-4159.
7. Prince A. The bitter taste of infection. $J$ Clin Invest. 2012;122:3847-3849.

8. Lee RJ, Cohen NA. The emerging role of the bitter taste receptor T2R38 in upper respiratory infection and chronic rhinosinusitis. Am J Rhinol Allergy. 2013;27:283-286.

9. Farquhar DR, Kovatch KJ, Palmer JN, Shofer FS, Adappa ND, Cohen NA. Phenylthiocarbamide taste sensitivity is associated with sinonasal symptoms in healthy adults. Int Forum Allergy Rhinol. 2015;5:111-118.

10. Shah AS, Ben-Shahar Y, Moninger TO, Kline JN, Welsh MJ. Motile cilia of human airway epithelia are chemosensory. Science. 2009;325:1131-1134.

11. Lim J, Urban L, Green BG. Measures of individual differences in taste and creaminess perception. Chemical Senses. 2008;33:493-501.

12. Mueller C, Kallert S, Renner B, et al. Quantitative assessment of gustatory function in a clinical context using impregnated "taste strips". Rhinology. 2003;41:2-6.

13. Mueller CA, Khatib S, Temmel AFP, Baumgartner WD, Hummel T. Effects of cochlear implantation on gustatory function. Ann Otol Rhinol Laryngol. 2007;116: 498-501.

14. Mueller CA, Khatib S, Naka A, Temmel AFP, Hummel T. Clinical assessment of gustatory function before and after middle ear surgery: a prospective study with a two-year follow-up period. Ann Otol Rhinol Laryngol. 2008;117:769773.

15. Mueller CA, Pintscher K, Renner B. Clinical test of gustatory function including umami taste. Ann Otol Rhinol Laryngol. 2011;120:358-362.

16. Wolf A, Illini O, Uy D, Renner B, Mueller CA. A new extension to the "taste strips" test. Rhinology. 2016;54:45-50.

17. Landis BN, Welge-Luessen A, Brämerson A. "Taste strips"a rapid, lateralized, gustatory bedside identification test based on impregnated filter papers. J Neurol. 2009;256: 242-248. 
18. Hayes JE, Feeney EL, Nolden AA, McGeary JE. Quinine bitterness and grapefruit liking associate with allelic variants in TAS2R31. Chem Senses. 2015;40:437-443.

19. Hummel T, Erras A, Kobal G. A test for the screening of taste function. Rhinology. 1997;35:146-148.

20. Ahne G, Erras A, Hummel T, Kobal G. Assessment of gustatory function by means of tasting tablets. Laryngoscope. 2000;110:1396-1401.

21. Lee RJ, Kofonow JM, Rosen PL, et al. Bitter and sweet taste receptors regulate human upper respiratory innate immunity. J Clin Invest. 2014;124:1393-1405.

22. Workman AD, Brooks SG, Kohanski MA, et al. Bitter and sweet taste tests are reflective of disease status in chronic rhinosinusitis [Published online October 17, 2017]. J Allergy Clin Immunol Pract. doi:10.1016/j.jaip.2017.09.01423

23. Lee RJ, Hariri BM, McMahon DB, et al. Bacterial d-amino acids suppress sinonasal innate immunity through sweet taste receptors in solitary chemosensory cells. Sci Signal. 2017;10(495).

24. Deems DA, Doty RL, Settle RG, et al. Smell and taste disorders, a study of 750 patients from the University of Pennsylvania Smell and Taste Center. Arch Otolaryngol Head Neck Surg. 1991;117:519-528.

25. Temmel AFP, Quint C, Schickinger-Fischer B, Klimek L, Stoller E, Hummel T. Characteristics of olfactory disorders in relation to major causes of olfactory loss. Arch Otolaryngol Head Neck Surg. 2002;128:635-641. 